\title{
RESEARCH CONCERNING THE STATIC BALANCING OF A \\ MULTILOOP LINKAGE
}

\section{Dorin Bădoiu ${ }^{1}$}

\author{
${ }^{1}$ Universitatea Petrol-Gaze din Ploieşti, România \\ e-mail: dorin.badoiu@gmail.com;
}

DOI: 10.51865/JPGT.2021.01.08

\begin{abstract}
In the paper a method for achieving the total static balancing of a linkage with two independent loops is presented. There are also analyzed two solutions for partial static balancing of the studied linkage. Finally, a series of results of the simulations performed in the studied balancing cases are given.
\end{abstract}

Keywords: static balancing, multiloop linkage, inertia forces

\section{INTRODUCTION}

The dynamic analysis of the mechanisms used in various applications, many of them even in the oil field, is a subject of study that is always up to date [1-7]. The problems that need to be solved in this case are generally of great complexity and have as aim finding of optimal solutions for the functioning of the mechanisms [8-14]. An important aspect related to the dynamics of the mechanisms is that of their balancing. This paper analyzes the static balancing of a linkage with two independent loops (Figure 1). First, a total static balancing solution of the linkage is presented and then two partial static balancing solutions are analyzed.

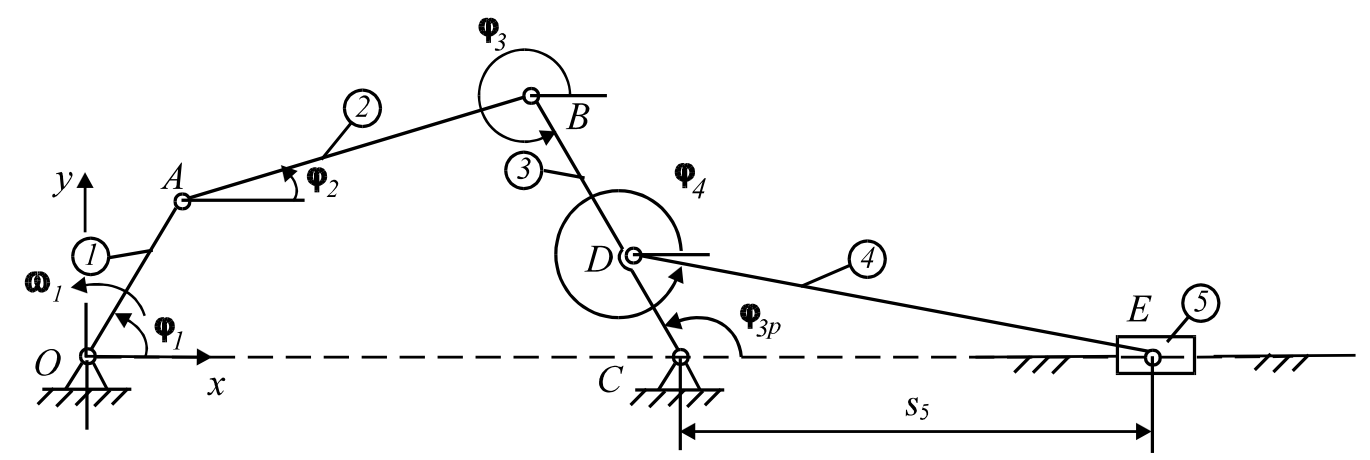

Figure 1. Linkage with two independent loops

\section{THEORETICAL CONSIDERATIONS AND SIMULATION RESULTS}

The graph associated [15] to the analyzed linkage is represented in figure 2 and it highlights the two independent loops: $0-1-2-3-0$ and $0-3-4-5-0$. 


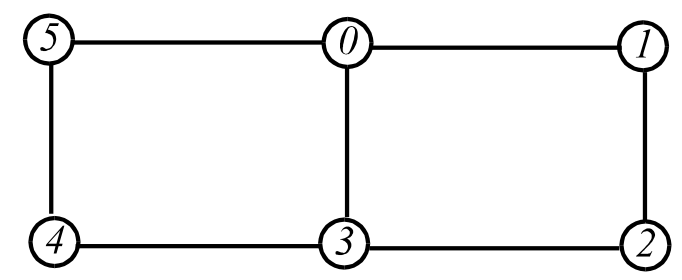

Figure 2. The graph associated to the analyzed linkage

By projecting the vector equations: $\overline{O A}+\overline{A B}+\overline{B C}+\overline{C O}=0$ and $\overline{C D}+\overline{D E}+\overline{E C}=0$ (Figure 1) corresponding to the component loops: 0-1-2-3-0 and 0-3-4-5-0 on the $x$ and $y$ axes, the following systems of equations are obtained:

$\left\{\begin{array}{l}l_{1} \cdot \cos \varphi_{1}+l_{2} \cdot \cos \varphi_{2}+l_{3} \cdot \cos \varphi_{3}-l_{0}=0 \\ l_{1} \cdot \sin \varphi_{1}+l_{2} \cdot \sin \varphi_{2}+l_{3} \cdot \sin \varphi_{3}=0\end{array}\right.$
$\left\{\begin{array}{l}l_{3 p} \cdot \cos \varphi_{3 p}+l_{4} \cdot \cos \varphi_{4}-s_{5}=0 \\ l_{3 p} \cdot \sin \varphi_{3 p}+l_{4} \cdot \sin \varphi_{4}=0\end{array}\right.$

where: $l_{1}=O A, l_{2}=A B, l_{3}=B C, l_{0}=C O, l_{3 p}=C D, l_{4}=D E$ and $\varphi_{3 p}=\varphi_{3}-\pi$.

By solving these systems of equations, the angles $\varphi_{2}, \varphi_{3}, \varphi_{4}$ and the displacement $s_{5}$ may be calculated with the next relations:

$A_{2} \cdot \cos \varphi_{2}+B_{2} \cdot \sin \varphi_{2}=C_{2}$

where:

$$
\begin{aligned}
& \left\{\begin{array}{l}
A_{2}=2 \cdot l_{1} \cdot l_{2} \cdot \cos \varphi_{1}-2 \cdot l_{0} \cdot l_{2} \\
B_{2}=2 \cdot l_{1} \cdot l_{2} \cdot \sin \varphi_{1} \\
C_{2}=l_{3}^{2}-l_{1}^{2}-l_{2}^{2}-l_{0}^{2}+2 \cdot l_{0} \cdot l_{1} \cdot \cos \varphi_{1}
\end{array}\right. \\
& \varphi_{3}=\operatorname{ATAN} 2\left(-\frac{1}{l_{3}}\left(l_{1} \cdot \sin \varphi_{1}+l_{2} \cdot \sin \varphi_{2}\right),-\frac{1}{l_{3}}\left(l_{1} \cdot \cos \varphi_{1}+l_{2} \cdot \cos \varphi_{2}-l_{0}\right)\right)
\end{aligned}
$$

where $\operatorname{ATAN} 2(y, x)$ calculates $\arctan (y / x)$ by taking into account the signs of $x$ and $y$.

$$
\begin{aligned}
& \sin \varphi_{4}=\frac{l_{3 p}}{l_{4}} \cdot \sin \varphi_{3} \\
& s_{5}=-l_{3 p} \cdot \cos \varphi_{3}+l_{4} \cdot \cos \varphi_{4}
\end{aligned}
$$

The angular speeds $\omega_{j}, j=2,3,4$ and the angular acceleration $\varepsilon_{j}, j=2,3,4$ are calculated with the relations:

$$
\omega_{j}=\dot{\varphi}_{j}=\frac{\mathrm{d} \varphi_{j}}{\mathrm{~d} \varphi_{1}} \cdot \frac{\mathrm{d} \varphi_{1}}{\mathrm{~d} t}=\omega_{1} \cdot \frac{\mathrm{d} \varphi_{j}}{\mathrm{~d} \varphi_{1}} ; j=2,3,4
$$


$\varepsilon_{j}=\dot{\omega}_{j}=\varepsilon_{1} \cdot \frac{\mathrm{d} \varphi_{j}}{\mathrm{~d} \varphi_{1}}+\frac{\mathrm{d}^{2} \varphi_{j}}{\mathrm{~d} \varphi_{1}^{2}} \cdot \omega_{1}^{2} ; j=2,3,4$

where $\omega_{1}$ is the angular speed of the crank 1 and $\varepsilon_{1}$ is its angular acceleration.

Once the angles $\varphi_{2}, \varphi_{3}$ and $\varphi_{4}$ established, the coordinates of any point on the linkage can be determined. The projections on the $x$ and $y$ axes of the speed and of the acceleration of any point $P$ belonging to the component elements of the linkage may be calculated by deriving in relation to time its coordinates:

$$
\begin{aligned}
& \left(v_{P}\right)_{x}=\dot{x}_{P}=\frac{\mathrm{d} x_{P}}{\mathrm{~d} \varphi_{1}} \cdot \frac{\mathrm{d} \varphi_{1}}{\mathrm{~d} t}=\omega_{1} \cdot \frac{\mathrm{d} x_{P}}{\mathrm{~d} \varphi_{1}} \\
& \left(v_{P}\right)_{y}=\dot{y}_{P}=\frac{\mathrm{d} y_{P}}{\mathrm{~d} \varphi_{1}} \cdot \frac{\mathrm{d} \varphi_{1}}{\mathrm{~d} t}=\omega_{1} \cdot \frac{\mathrm{d} y_{P}}{\mathrm{~d} \varphi_{1}} \\
& \left(a_{P}\right)_{x}=\ddot{x}_{P}=\varepsilon_{1} \cdot \frac{\mathrm{d} x_{P}}{\mathrm{~d} \varphi_{1}}+\frac{\mathrm{d}^{2} x_{P}}{\mathrm{~d} \varphi_{1}^{2}} \cdot \omega_{1}^{2} \\
& \left(a_{P}\right)_{y}=\ddot{y}_{P}=\varepsilon_{1} \cdot \frac{\mathrm{d} y_{P}}{\mathrm{~d} \varphi_{1}}+\frac{\mathrm{d}^{2} y_{P}}{\mathrm{~d} \varphi_{1}^{2}} \cdot \omega_{1}^{2}
\end{aligned}
$$

The static balancing of the mechanisms aims to reduce the value of the resultant of all inertia forces acting on the component elements. This leads to the reduction of the level of vibrations and shocks during operation and to the reduction of the wear of the connecting joints between the elements. On the other hand, static balancing is achieved by mounting balancing masses in the extension of the elements of the mechanisms, which leads to an increase in their gauge. Therefore, the solutions of partial static balancing of the mechanisms that lead to the significant reduction of the values of the inertia forces in the conditions of an insignificant increase of the gauge are indicated.

A solution for total static balancing of the linkage is presented in Figure 3, when the resultant of all the inertia forces acting on the component elements is cancelled. A model with concentrated masses [16] was used in which:

$$
\left\{\begin{array}{l}
m_{O}=m_{1 O} \\
m_{A}=m_{1 A}+m_{2 A} \\
m_{B}=m_{2 B}+m_{3 B} \\
m_{C}=m_{3 C} \\
m_{D}=m_{4 D} \\
m_{E}=m_{4 E}+m_{5}
\end{array}\right.
$$

where: $m_{5}$ is the mass of the piston 5 and by considering that the mass centres $C_{j}, j=1,2,3,4$, are at the middle of the corresponding elements it results that the concentrated masses $m_{1 O}$ and $m_{1 A}$ are equal to half of the mass $m_{1}$ of element $1, m_{2 A}$ 
and $m_{2 B}$ are equal to half of the mass $m_{2}$ of element $2, m_{3 B}$ and $m_{3 C}$ are equal to half of the mass $m_{3}$ of element 3 and $m_{4 D}$ and $m_{4 E}$ are equal to half of the mass $m_{4}$ of element 4.

For the total static balancing of the linkage, the balancing mass $m_{E 1}$ was first added in the extension of the element 4 so that:

$m_{E 1} \cdot r_{E 1}=m_{E} \cdot l_{4}$

From equation (15) the balancing mass $m_{E 1}$ and the length of its arm $r_{E 1}$ are calculated by imposing the value of one of the two unknowns.

By neglecting the mass of the arm of length $r_{E 1}$, it results that in point $D$ a mass equal to $M_{D}=m_{E 1}+m_{D}+m_{E}$ is now concentrated.

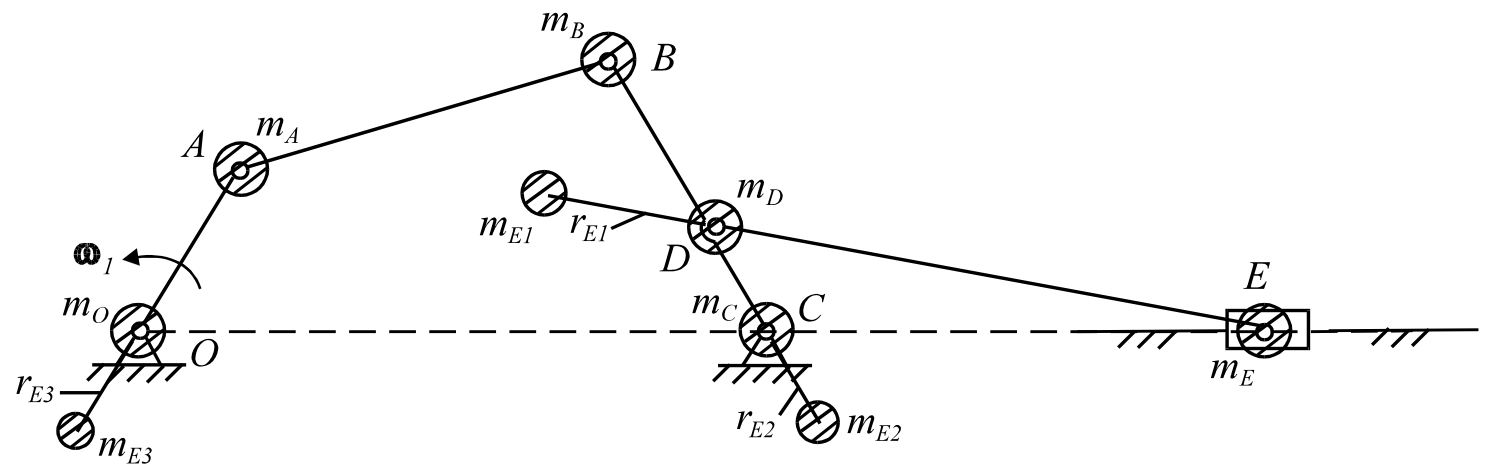

Figure 3. Solution for total static balancing of the analyzed linkage

Then, the balancing mass $m_{E 2}$ is added in the extension of the rocker arm 3 so that:

$m_{E 2} \cdot r_{E 2}=m_{B} \cdot l_{3}+M_{D} \cdot l_{3 p}$

The balancing mass $m_{E 2}$ and the length of its arm $r_{E 2}$ are calculated in the same way, by imposing the value of one of them. Neglecting the mass of the arm of length $r_{E 2}$, it results that in point $C$ is now concentrated a mass equal to $M_{C}=m_{E 2}+M_{D}+m_{B}$.

Finally, the balancing mass $m_{E 3}$ in the extension of the crank 1 is added, so that:

$m_{E 3} \cdot r_{E 3}=m_{A} \cdot l_{1}$

After setting the values for $m_{E 3}$ and $r_{E 3}$ by imposing the value of one of them in equation (17), it results (by neglecting the mass of the arm of length $r_{E 3}$ ) that in point $O$ is concentrated a mass equal to $M_{O}=m_{E 3}+m_{O}+m_{A}$.

A computer program that simulates the functioning of the linkage has been realized using Maple programming environment. It was considered that the elements of the 
linkage are made of bars of aluminium having the following radii: $0.02 \mathrm{~m}$ for the crank $1,0.025 \mathrm{~m}$ for the elements 2 and 3 and $0.03 \mathrm{~m}$ for the element 4 . The mass of the piston 5 is of $3.5 \mathrm{~kg}$. The angular speed of the crank $\omega_{1}$ is equal to $20 \mathrm{rad} / \mathrm{s}$.

The following dimensions of the component elements of the linkage has been considered: $l_{1}=0.2 \mathrm{~m} ; l_{2}=0.5 \mathrm{~m} ; l_{3}=0.55 \mathrm{~m}, l_{0}=0.6 \mathrm{~m}, l_{3 p}=0.2 \mathrm{~m}$ and $l_{4}=0.8 \mathrm{~m}$.

In the case of the total static balancing of the analyzed linkage, the lengths of the arms of the balancing masses has been imposed in the following way: $r_{E 1}=0.4 \cdot l_{4}$, $r_{E 2}=0.4 \cdot l_{3}$ and $r_{E 3}=0.8 \cdot l_{1}$. The balancing masses have resulted with the following values: $m_{E 1}=16.384 \mathrm{~kg}, m_{E 2}=30.586 \mathrm{~kg}$ and $m_{E 3}=2.08 \mathrm{~kg}$.

Due to the increase of the mechanism gauge, especially by adding the balancing mass $m_{E 1}$ and due to the high values of the balancing masses $m_{E 1}$ and $m_{E 2}$, partial balancing solutions were sought.

Figure 4 shows a first partial balancing solution in which the balancing mass $m_{E 1}$ was abandoned, keeping the balancing masses $m_{E 2}$ and $m_{E 3}$. In this case, by keeping the lengths of the arms of these balancing masses $r_{E 2}=0.4 \cdot l_{3}$ and $r_{E 3}=0.8 \cdot l_{1}$, $m_{E 2}=9.734 \mathrm{~kg}$ has been obtained.

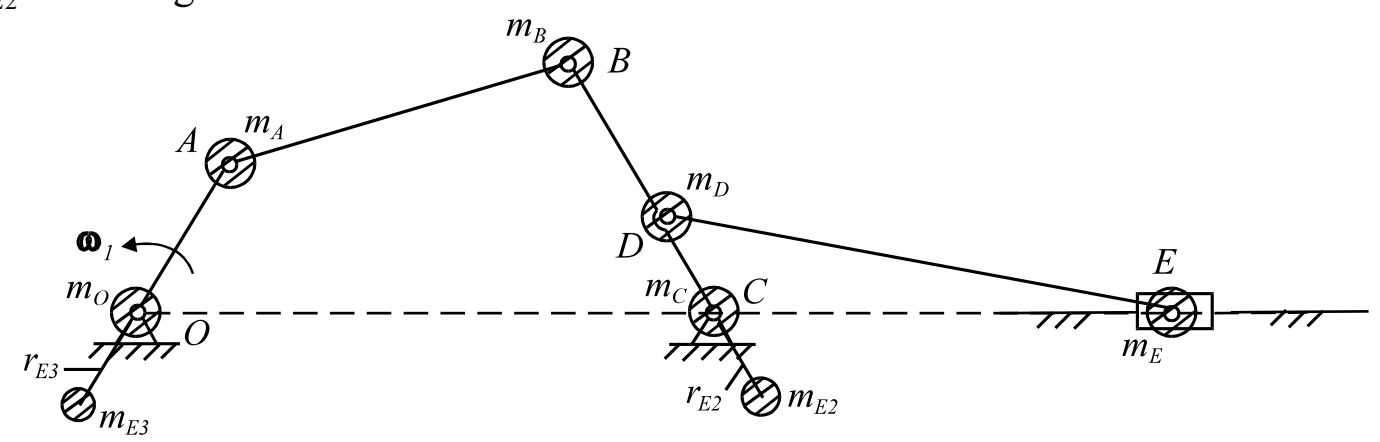

Figure 4. First solution for partial static balancing of the analyzed linkage

In Figure 5 it is presented a second partial balancing solution in which the balancing mass $m_{E 2}$ is supplemented with the mass $m_{E s}$ whose expression is: $m_{E s}=m_{E} \cdot l_{3 p} / r_{E 2}$, so that the horizontal component of the inertia force developed by it balances the first harmonic of the inertia force developed by the mass $m_{E}$ [16].

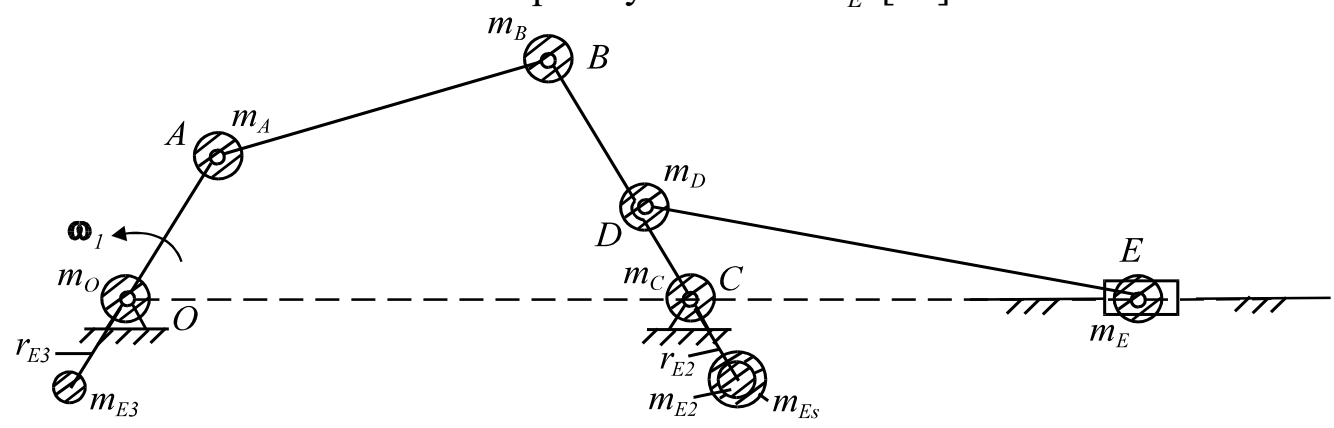

Figure 5. Second solution for partial static balancing of the analyzed linkage 
In Figure 6 it is presented the variation on a cinematic cycle of the total inertia force $F_{\text {itot }}$ in the case when the linkage is unbalanced (curve 1 ) and when it is partial balanced (curve 2 for the first solution of partial balancing and curve 3 when is added the mass $m_{E S}$ ). The total inertia force $F_{i t o t}$ has been calculated by considering its projections on the $x$ and $y$ axes with the relation: $F_{\text {itot }}=\sqrt{F_{\text {itotx }}^{2}+F_{\text {itoty }}^{2}}$, where:

$$
\left\{\begin{array}{l}
F_{i t o t x}=-\sum_{j=1}^{5} m_{j} \cdot\left(a_{C j}\right)_{x} \\
F_{\text {itoty }}=-\sum_{j=1}^{5} m_{j} \cdot\left(a_{C j}\right)_{y}
\end{array}\right.
$$

when the linkage is unbalanced, and:

$$
\begin{aligned}
& \left\{\begin{array}{l}
F_{\text {itotx }}=-\sum_{j=1}^{5} m_{j} \cdot\left(a_{C j}\right)_{x}-\left(m_{E 2} \cdot a_{E 2 x}+m_{E 3} \cdot a_{E 3 x}\right) \\
F_{\text {itoty }}=-\sum_{j=1}^{5} m_{j} \cdot\left(a_{C j}\right)_{y}-\left(m_{E 2} \cdot a_{E 2 y}+m_{E 3} \cdot a_{E 3 y}\right)
\end{array}\right. \\
& \left\{\begin{array}{l}
F_{\text {itotx }}=-\sum_{j=1}^{5} m_{j} \cdot\left(a_{C j}\right)_{x}-\left(\left(m_{E 2}+m_{E s}\right) \cdot a_{E 2 x}+m_{E 3} \cdot a_{E 3 x}\right) \\
F_{\text {itoty }}=-\sum_{j=1}^{5} m_{j} \cdot\left(a_{C j}\right)_{y}-\left(\left(m_{E 2}+m_{E s}\right) \cdot a_{E 2 y}+m_{E 3} \cdot a_{E 3 y}\right)
\end{array}\right.
\end{aligned}
$$

for the two cases of partial balancing. In relations (18), (19) and (20), $a_{C j}$ represents the acceleration of the mass centre $C_{j}$ of the element $j$ and $a_{E 2}$ and $a_{E 3}$ represent the accelerations of the points where the corresponding balancing masses are concentrated.

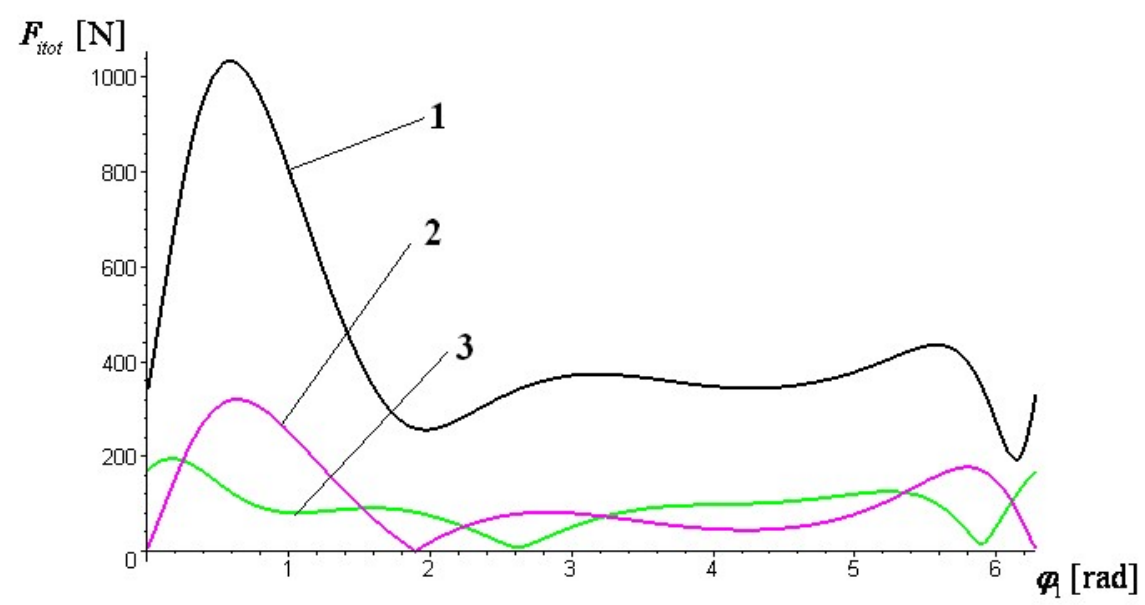

Figure 6. The variation on a cinematic cycle of the total inertia force $F_{\text {itot }}$ in the case when the linkage is unbalanced (curve 1) and when it is partially balanced (curves 2 and 3) 


\section{CONCLUSIONS}

In the paper a method for achieving the total static balancing of a linkage with two independent loops has been presented. There has also been presented two solutions for partial static balancing of the analysed linkage. The results of the simulations showed a significant decrease in the value of the resultant of the inertia forces in the two cases of partial static balancing, especially in the case when the balancing mass $m_{E 2}$ has been supplemented with the mass $m_{E s}$ (Figure 5). Taking into account the important reduction of the mechanism gauge in the two cases of partial balancing compared to the case of total static balancing, it results that from a practical point of view it is more indicated to use the two analysed partial balancing configurations.

\section{REFERENCES}

[1] Bădoiu D., Toma G., Research on designing a multiloop planar linkage, IOP Conference Series: Materials Science and Engineering, Vol. 659, Issue 1, pp. 1-9, 2019.

[2] Bădoiu D., Toma G., Analysis of the dynamic response of the mechanism of conventional sucker rod pumping units, Revista de Chimie, Vol. 71, Issue 1, pp. 395399, 2020.

[3] Toma G., Research concerning the optimization of the mechanism of the conventional sucker rod pumping units, Revista de Chimie, Vol. 70, Issue 5, pp. 17951799, 2019.

[4] Toma G., Research concerning the dynamic model of the conventional sucker rod pumping units, Revista de Chimie, Vol. 70, Issue 8, pp. 2818-2821, 2019.

[5] Bădoiu D., Toma G., Research concerning the predictive evaluation of the motor moment at the crankshaft of the conventional sucker rod pumping units, Revista de Chimie, Vol. 70, Issue 2, pp. 378-381, 2019.

[6] Bădoiu D., Toma G., Research concerning the kinetostatic analysis of the mechanism of the conventional sucker rod pumping units, Revista de Chimie, Vol. 69, Issue 7, pp. 1855-1859, 2018.

[7] Bădoiu D., Toma G., Research concerning the correlations between some experimental results in the case of a sucker rod pumping installation, Revista de Chimie, Vol. 69, Issue 11, pp. 3060-3063, 2018.

[8] Toma G., Bădoiu D., On the variation of the motor moment in the case of a total statically balanced quadrilateral mechanism, Petroleum-Gas University of Ploiesti Bulletin, Technical Series, Vol. 69, Issue 1, pp. 69-74, 2017.

[9] Bădoiu D., Toma G., Research concerning the identification of some parameters of a sucker rod pumping unit, Revista de Chimie, Vol. 68, Issue 10, pp. 2289-2292, 2017.

[10] Toma G., Pupăzescu A., Research concerning the functional constructive optimization of the sucker rod pumping units, Petroleum-Gas University of Ploiesti Bulletin, Technical Series, Vol. 68, Issue 2, pp. 67-72, 2016. 
[11] Bădoiu D., Toma G., On a dynamic optimisation problem of the quadrilateral mechanism, Journal of the Balkan Tribological Association, Vol. 22, Issue 1, pp. 250$260,2016$.

[12] Toma G., Bădoiu D., On the cinematic analysis of a mechanism of fourth class, Petroleum-Gas University of Ploiesti Bulletin, Technical Series, Vol. 64, Issue 1, pp. 69-72, 2012.

[13] Bădoiu D., Toma G., Research concerning the optimization of a mechanism with two independent contours, Romanian Journal of Petroleum \& Gas Technology, Vol. I (LXXII), Issue 2, 2020.

[14] Toma G., Bădoiu D., Research concerning the dynamic analysis of a crank and connecting rod mechanism, Romanian Journal of Petroleum \& Gas Technology, Vol. I (LXXII), Issue 2, 2020.

[15] Bădoiu D., Toma G., Structura si cinematica mecanismelor cu bare, Editura Universităţii Petrol-Gaze din Ploieşti, Ploieşti, 2019.

[16] Bădoiu D., Analiza dinamica a mecanismelor si masinilor, Editura Didactica si Pedagogica, Bucuresti, 2003. 